\title{
Electron Counting Mode with Fiber-Optically Coupled Camera System
}

\author{
Reza Ghadimi ${ }^{1}$, Dominic Tietz ${ }^{1}$, Marco Oster ${ }^{1}$, Andreas Wisnet ${ }^{1}$ and Hans Tietz ${ }^{1}$ \\ 1. TVIPS GmbH, Gauting, Germany
}

Former scintillator-based CMOS camera systems with a classical 3 transistor pixel design already proved their usefulness several years ago [1]. Recent improvements in signal clarity for low dose applications with direct detecting camera systems can be mainly attributed to the use of drift correction and counting mode acquisition [2]. For certain applications, as low voltage microscopy [3] and imaging conditions with very high beam current densities, even last-generation fiber-optically coupled camera systems provide most favourable imaging properties [4]. We report on the advances of our latest CMOS camera system with a large field of view of $63.5 \times 63.5 \mathrm{~mm}^{2}$ structured in $4 \mathrm{k} \times 4 \mathrm{k}$ pixels, which are implemented in a state-of-the-art 4 Transistor Pixel Design with a Floating Diffusion node for on-chip Correlated Double Sampling (CDS) and an additional buried capacitance for on-demand extension of the intrinsic dynamic range.

For drift correction and counting mode acquisition, the essential requirements are a high frame rate and of course single electron sensitivity. To achieve both features at the same time, on-chip CDS is mandatory. On-chip CDS results in superior signal-to-noise ratio $(\mathrm{S} / \mathrm{N})$ due to the excellent time correlation of reset and image signal and is also beneficial for reading out the sensor rapidly.

We could achieve full sensor readout with 48 frames per second (fps) and $380 \mathrm{fps}$ for subareas. The sensitivity is sufficient to detect single electron events (SEE) for acceleration voltages from $20 \mathrm{kV}$ to $400 \mathrm{kV}$ (cf. Figure 1).

Compared to direct detectors, fiber-optically coupled camera systems can offer a much larger intrinsic full well capacity, resulting in a better capability of faithfully recording large intensity differences in a single acquisition (integration or linear mode) with only one readout and hence minimized readout noise. The large field of view, high sensitivity and intrinsic high dynamic range therefore render the system an ideal detector for high-resolution diffraction experiments like MicroED. Advanced diffraction experiments like precessed 4D-STEM are enabled by the possibility of modulating the beam and reading out the camera synchronously (cf. Figure 2).

\section{References:}

[1] M Stumpf et al., Microsc. Microanal. 16(S2) (2010), p. 856.

[2] McMullan et al., in "Methods in Enzymology Vol. 579", p. 1.

[3] C Kealhofer et al., Ultramicroscopy 159 (2015), p. 19.

[4] JA Rodriguez and T Gonen in "Methods in Enzymology Vol. 579", p. 369. 

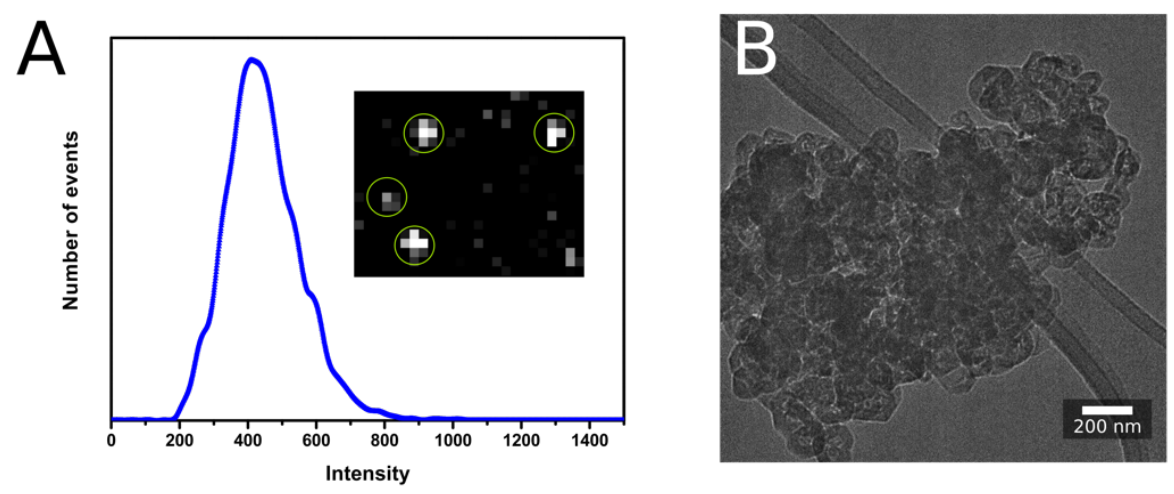

Figure 1. Histogram of the integrated intensities around SEEs at $80 \mathrm{kV}$ (A). The inset shows several detected SEEs. Reconstructed image of a graphite specimen probed by 19 million electrons at an acceleration voltage of $120 \mathrm{kV}(\mathrm{B})$.
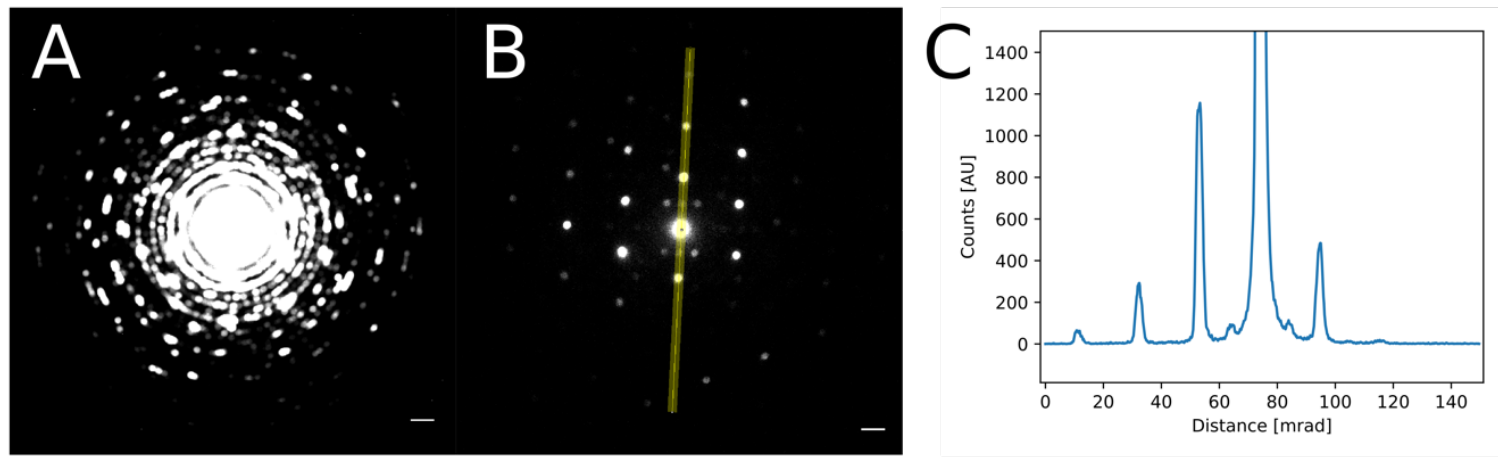

Figure 2. Precessed CBED patterns from a mapping experiment. (A) shows the average diffraction pattern for all 10000 scan positions with the contrast scaled such that the high angle intensities are visible. (B) shows the .iffraction pattern of a single scan position. (C) shows the dynamic of the line scan data from the region depicted in (B). Scale bars are $10 \mathrm{mrad}$. 